\title{
Sodium Salicylate Activates Cathepsin B But Not Cathepsin H from Rat Spleen
}

\author{
Kenji YAMAMOTO, Mitsue TAKEDA and Yuzo KATO \\ Department of Pharmacology. Nagasaki University School of Dentistry. Nagasaki 852. Japan
}

Accepted March 18, 1985

\begin{abstract}
Effect of sodium salicylate (SA) and its analogues on lysosomal cysteine proteinases, cathepsins $\mathrm{B}$ and $\mathrm{H}$. was investigated. Using a sensitive fluorometric assay. SA was shown to activate cathepsin B but not cathepsin $\mathrm{H}$ in the presence of cysteine. Kinetics data showed that the SA-stimulated reaction was due to a 4.5 fold decrease in $K_{m}$. SA caused a decrease in the inactivation rate of cathepsin $B$ by leupeptin and E-64. It was suggested that SA stimulates the activation process of the essential cysteine residue.
\end{abstract}

Cathepsins $B$ and $H$ are different but analogous lysosomal cysteine proteinases (1-3). Despite their physiological and pathological roles in protein catabolism $(2,3)$, we do not know the exact part played by each enzyme. We also lack the information on their natural substrates and conditions of action in vivo. Therefore, our knowledge of the significance of the existence of these analogous enzymes is still incomplete and much work is required. For this, we need detailed information on the catalytic-site characteristics of each enzyme and on effects of the structural change on the catalytic properties. Recently, the catalytic-site properties of these enzymes have been demonstrated by using some selective protease inhibitors (2-5)

In a previous work (6). we demonstrated that cathepsin B could be distinguished from cathepsin $\mathrm{H}$ by differences in the effect of flufenamic acid and indomethacin on their catalytic properties. In this communication, we describe the selective activation of cathepsin B. but not cathepsin H. by sodium salicylate and studied the nature and extent of the SA-stimulated reaction.

Cathepsins $B$ and $H$ were purified from rat spleen as described $(4,7)$. Since rat spleen cathepsins $B$ and $H$ consist of two and three isoenzymes, respectively, the enzymes used in this study were the respective major forms (B, form 1: $H$, form II). The activities of cathepsins $B$ and $H$ were measured fluorometrically by using benzyloxycarbonyl-phenylalanyl-arginine-4-methyl-7coumarylamide (Z-Phe-Arg-MCA) and arginine-4-methyl-7-coumarylamide (ArgMCA) as the respective substrates according to the method of Barrett (8), with a modification as described (6). The molar concentration of solutions of these two enzymes was determined by inactivation with E-64, N-[N-(L-3-trans-carboxyoxirane-2carbonyl) -L-leucyl] -agmatine (9).

Table 1 shows the effect of SA and its analogues on cathepsins $B$ and $H$. The enzyme concentrations were kept constant in all experiments $\left(B, 2.0 \times 10^{-7} \mathrm{M} ; \mathrm{H}\right.$, $\left.1.6 \times 10^{-6} \mathrm{M}\right)$. Of the three monohydroxy benzoates, the ortho isomer (SA) alone activated cathepsin $B$, but not cathepsin $H$, in a dose-dependent manner. The meta and para isomers showed little or no effect on both enzymes. Aspirin did not affect activities of these two enzymes. The selectivity and activating potency of SA for cathepsin B is probably due to its high binding capacity for the molecule, since the presence of a hydrogen donor in the ortho position conferred greater binding ability for proteins than the same radicals in the meta and para positions (10). The same results were obtained for rat liver cathepsins $\mathrm{B}$ and $\mathrm{H}$ (not shown). As Takio et al. (11) reported that the primary structure of the catalytic site 
Table 1. Effects of sodium salicylate and ts analogues on purified cathepsins $B$ and $H$ from rat spleen

\begin{tabular}{lcrr}
\hline & Concentration & \multicolumn{2}{c}{ \% of activity } \\
Compound & $(\mathrm{M})$ & Cathepsin B & Cathepsin H \\
Control & & 100 & 100 \\
Acetylsalicylic acid & $5.6 \times 10^{-5}$ & 100 & 94 \\
& $5.6 \times 10^{-4}$ & 98 & 92 \\
0-Hydroxybenzoate (SA) & $5.6 \times 10^{-3}$ & 94 & 97 \\
& $6.2 \times 10^{-5}$ & 92 & 90 \\
m-Hydroxybenzoate & $6.2 \times 10^{-4}$ & 120 & 95 \\
& $6.2 \times 10^{-3}$ & 240 & 100 \\
p-Hydroxybenzoate & $6.2 \times 10^{-5}$ & 96 & 99 \\
& $6.2 \times 10^{-4}$ & 94 & 98 \\
& $6.2 \times 10^{-3}$ & 92 & 100 \\
\hline
\end{tabular}

The purified cathepsins $B\left(2.0 \times 10^{-7} \mathrm{M}\right)$ and $\mathrm{H}\left(1.6 \times 10^{-6} \mathrm{M}\right)$ were preincubated with each compound at the indicated concentration in the respective assay buffers containing $5 \mathrm{mM}$ cysteine and $1 \mathrm{mM}$ EDTA at $25^{\circ} \mathrm{C}$ for $10 \mathrm{~min}$. The residual activities were measured by using the rospective substrates and expressed as percentages of the respective controis which were assayed in the absence of each drug.

Table 2. Effects of activators and inhibitors on cathepsins $B$ and $H$ from rat spleen

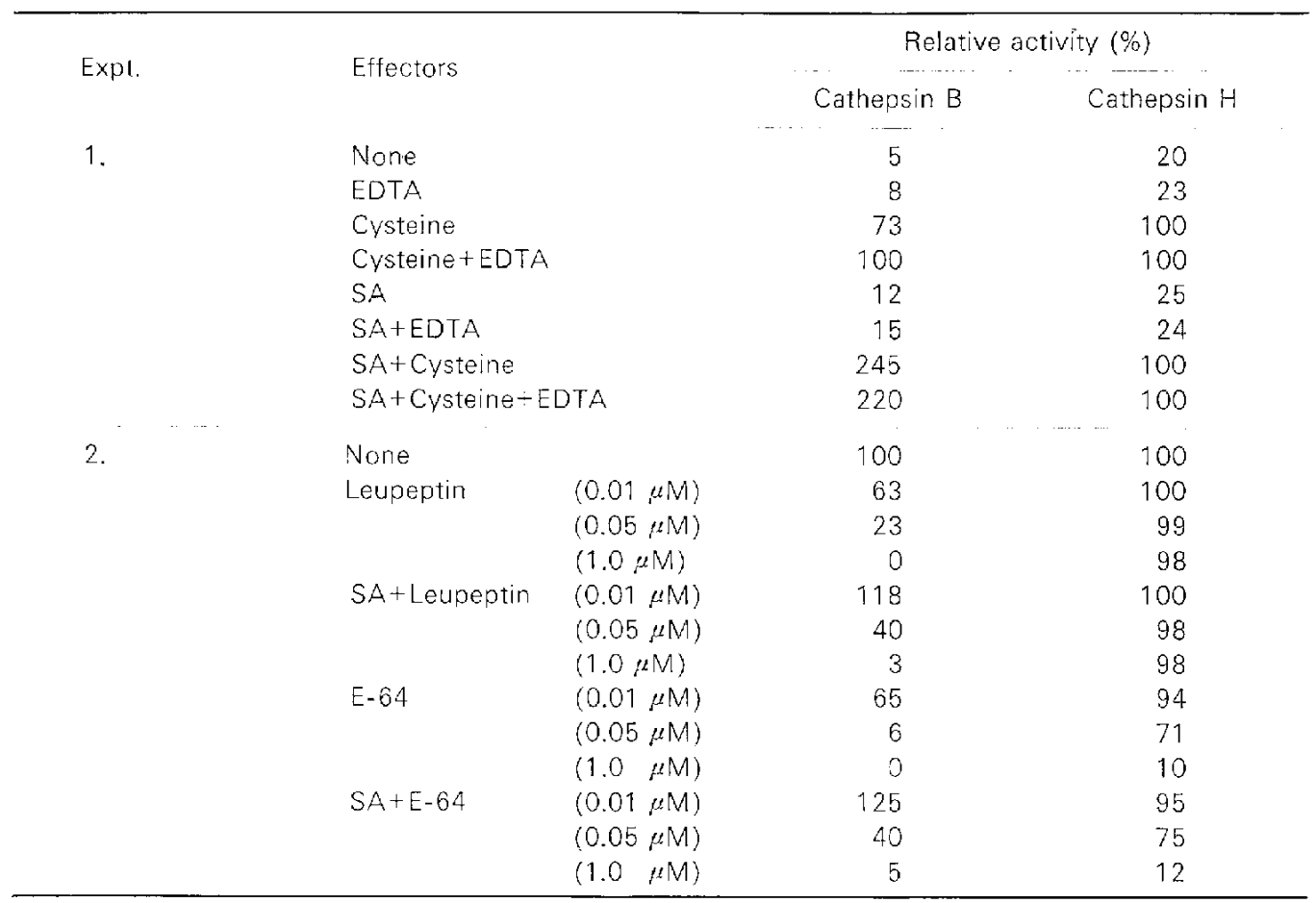

Final concentrations of cysteine, EDTA and SA were $5 \mathrm{mM}, 1 \mathrm{mM}$ and $6.2 \mathrm{mM}$, respectively. In experiment 2. cathepsins $B$ and $H$ in the respective assay buffers containing cysteine and EDTA were preincubated at $\mathrm{O}^{\circ} \mathrm{C}$ for $5 \mathrm{~min}$ in the presence or in the absence of SA prior to the 5 -min incubation with leupeptin or E-64 at $30^{\circ} \mathrm{C}$. The residual activities were measured by using the respective methylcoumarylamide substrates. 
region of rat liver cathepsin $B$ was closely similiar to that of cathepsin $\mathrm{H}$, the discrepancy in the effect of SA on these two enzymes is of particular interest. Cysteine was essential for the SA-stimulated reaction. while EDTA is not necessarily required (Table 2). No significant activation of cathepsin B was observed by SA alone or by SA plus EDTA. Other thiol-activating compounds such as dithiothreitol, 2-mercaptoethanol and reduced glutathione mediated the same SA-stimulated reaction for cathepsin $B$ as cysteine did. Kinetic experiments were performed on rat spleen cathepsin B at $\mathrm{pH} 6.0$, varying the substrate concentrations (5 $/ \mathrm{M}-320 / \mathrm{M})$ in the presence of $5 \mathrm{mM}$ cysteine and $1 \mathrm{mM}$ EDTA. The $K_{m}$ value of cathepsin $B$ was $286 / \mathrm{M}$, which is consistent with that of cathepsin B from human liver (8) and porcine kidney (12). Comparison of Lineweaver-Burk plots of control and SAtreated cathepsin $B$ indicated that the SAstimulated reaction was due to a 4.5 -fold decrease in $K_{m}$. About a 2.4-fold activation of cathepsin $B$ was observed under the standard assay conditions using $10 \% \mathrm{M}$ substrate, but SA resulted in only $20 \%$ activation of cathepsin B when the substrate concentrations were equal to approximately the $K_{m}$ value. This may reflect the fact that the SAstimulated reaction was accompanied by about a 2 -fold decrease in $V_{\max }$.

The inactivation rate of cathepsin $B$ by leupeptin and E-64, which are known as active-site-directed inhibitors, was significantly decreased by preincubation with SA prior to the addition of each inhibitor (Table 2). Both leupeptin and E-64 strongly inhibited cathepsin B, but E-64 had a greater effect than leupeptin on cathepsin $H$. Since the selectivity and potency of these compounds depend on their affinities for the proteinases. the decrease in the inactivation rate of cathepsin B by these inhibitors appears to be due to the inhibitory effect of SA on the binding capacity of the inhibitors for the specificity sites of the enzyme. Therefore, the present data suggest that the activation of cathepsin B by SA under the assay conditions employed was due to the binding of SA to at least in part the catalytic site region. Since the activation of the essential cysteine residue of cathepsin $B$ by thiol-activating reagents such as dithiothreitol has been shown to be a slow process (13), the conformational change induced by SA-binding appears to stimulate this activation process of the enzyme. Although the physiological relevance of selective activation of cathepsin $B$ by $S A$ is not clear, the present results may offer some information on the pathological significance of structurally different analogous proteinases.

\section{References}

1 Kominami, E. and Katunuma, N.: Immunological studies on cathepsins $B$ and $H$ from rat liver. $J$. Biochem. 91, 67-71 (1982)

2 Barrett, A.J.: Cathepsin B and other thiol proteinases. In Proteinases in Mammalian Cells and Tissues. Edited by Barrett, A.J., p. 181-208, North-Holland Publishing Co., Amsterdam (1977)

3 Kirschke, H., Langner, J., Riemann, B., Wiederanders, S., Ansorge, S. and Bohley, P.: Lysosomal cysteine proteinases. In Protein Degradation in Health and Disease, p. 15-35. Ciba Foundation Symposium 75, Excerpta Medica, Amsterdam, Oxford and New York (1980)

4 Yamamoto, K., Kamata, 0 . and Kato, $Y$ : Separation and properties of three forms of cathepsin $H$-like cysteine proteinase from rat spleen. J. Biochem. 95, 477-484 (1984)

5 Willenbrock, F. and Brocklehurst, K.: Natural structural variation in enzymes as a tool in the study of mechanism exemplified by a comparison of catalytic-site structure and characteristics of cathepsin B and papain. Biochem. J. 222, 805$814(1984)$

6 Yamamoto, K, Kamata, O. and Kato, Y.: Differential effects of anti-inflammatory agents on |ysosomal cysteine proteinases cathepsins B and $\mathrm{H}$ from rat spleen. Japan. J. Pharmacol. 35, 253$258(1984)$

7 Yamamoto, K., Kamata, O., Takeda, M. and Kato, Y.: Characterization of rat spleen cathepsin $B$ and differential effects of anti-inflammatory drugs on its activity. Japan. J. Oral Bicl. 25,834838 (1983)

8 Barrett, A.J.: Fluorimetric assays for cathepsin B and cathepsin $H$ with methylcoumarylamide substrates. Biochem. J. 187, 909-912 (1980)

9 Barrett, A.J., Kembhavi, A.A., Brown, M.A., Kirschke, H., Knight, C.G., Tamai, M. and Hanada, K: L-trans-Epoxysucciny! leucylamido (4-guanidino)butane (E-64) and its analogues 
as inhibitors of cysteine proteinases including cathepsins B, H and L. Biochem. J. 201, 189198 (1982)

10 Davison, C. and Smith, P.K.: The binding of salicylic acid and related substances to purified proteins. J. Pharmacol. Exp. Ther. 133, $161 \ldots 170$ (1961)

11 Takio, K., Towatari, T., Katunuma, N., Teller, D.C. and Titani, K.: Homology of amino acid sequences of rat liver cathepsins $B$ and $H$ with that of papain. Proc. Natl. Acad. Sci, U.S.A. 80 , 3666-3670 (1983)

12 Takahashi, S., Murakami, K. and Miyake, Y.: Purification and characterization of porcine kidney cathepsin B. J. Biochem. 90, 1677-1684 (1981)

13 Baici, $A$, and Gyger-Marazzi, $M$ : The slow, tight-binding inhibition of cathepsin $B$ by leupeptin. A hysteretic effect. Eur. J. Biochem. $129,33-41(1982)$ 